\title{
Electron-Electron Momentum Exchange in Strong Field Double Ionization
}

\author{
M. Weckenbrock, ${ }^{1}$ A. Becker, ${ }^{2, *}$ A. Staudte, ${ }^{1}$ S. Kammer, ${ }^{1}$ M. Smolarski, ${ }^{1}$ V. R. Bhardwaj, ${ }^{3}$ D. M. Rayner, ${ }^{3}$ \\ D. M. Villeneuve, ${ }^{3}$ P. B. Corkum, ${ }^{3}$ and R. Dörner ${ }^{1, \dagger}$ \\ ${ }^{1}$ Institut für Kernphysik, Universität Frankfurt, D-60486 Frankfurt, Germany \\ ${ }^{2}$ Max-Planck-Institut für Physik komplexer Systeme, Nöthnitzer Strasse 38, D-01187 Dresden, Germany \\ ${ }^{3}$ National Research Council, 100 Sussex Drive, Ottawa, Ontario, Canada K1A OR6
}

(Received 4 April 2003; published 19 September 2003)

\begin{abstract}
We have investigated the momentum balance between the two electrons from strong field double ionization of argon at $780 \mathrm{~nm}$ and $1.9 \times 10^{14} \mathrm{~W} / \mathrm{cm}^{2}$. Experimental data show that perpendicular to the laser polarization direction the electrons emerge preferentially in opposite directions. Results of model calculations are found to agree well with the data and reveal a dominant role of the Coulomb correlation between the two outgoing electrons in this kinematical geometry. Differences between the experimental observations and the theoretical results for the ion momentum distribution indicate the importance of the further effects during the three-body breakup.
\end{abstract}

DOI: 10.1103/PhysRevLett.91.123004

The correlated motion of three-body Coulomb systems provides a major theoretical challenge in quantum mechanics and has far-reaching practical importance in many fields of physics and chemistry. One of the prominent processes, in which such correlated motion can be studied, is the double ejection of electrons from an atom in a strong laser field. Here, the basic three-body problem is closely interrelated with the highly nonlinear electronfield interaction. Two central questions in strong field double ionization are as follows: What is the mechanism by which the intense-field sets both electrons free, and what is the quantum dynamics of the particles in the light field once they are in the continuum?

A widely accepted consensus today (see [1,2] for reviews) is that the rescattering process $[3,4]$ provides the basic mechanism for double ionization at intermediate intensities $\left(<5 \times 10^{14} \mathrm{~W} / \mathrm{cm}^{2}\right.$ for Ar). It has been shown [5] that the rescattering mechanism corresponds to the leading Feynman diagram of a systematic quantum mechanical $S$-matrix analysis of strong field double ionization in the semiclassical limit. In this picture, the first electron is ionized by the field, accumulates energy during about half a laser circle, and is driven back to its parent ion where it assists with its energy in setting the second electron free. In particular, the later step of electron impact ionization in the presence of a strong field bares many open questions. How does the electron exchange its energy and its momentum with the ion and the second electron to be emitted? What is the role of the field in this process? How do the Coulomb interactions influence the dynamics of the two emerging electrons? These questions can be addressed by investigating the final-state momenta of the two electrons.

Experimental and theoretical studies on the electron and the recoiling ion momenta have mostly concentrated on the momentum components of the electrons and the ion in the polarization direction of the field [2]. These momenta are found to be dominated by the interaction of
PACS numbers: $32.80 . \mathrm{Rm}, 31.90 .+\mathrm{s}, 32.80 . \mathrm{Fb}, 32.80 . \mathrm{Wr}$

the particles with the strong external field after the electron impact ionization. The data can therefore be used to obtain information on the time evolution of the ionization process with subfemtosecond time resolution. A double peak structure in the parallel component of the ion momentum [6,7] gave direct evidence for the time delay introduced by the rescattering process. Further, both electrons have been found to be emitted predominantly to the same half sphere with similar parallel momenta [8]. This indicates that often both electrons are set free with small momentum close to the zero crossing of the oscillating field. In this case they both accumulate the same high parallel drift momentum [9-12].

In this Letter, we present a joint experimental and theoretical study of the widely unexplored momentum balance in the direction perpendicular to the laser field. The virtue of this new perspective on the process is that the perpendicular momenta are solely a product of the three-body interaction and not of the laser field. Thus, the subtleties of momentum exchange in the recollision process should not be covered by the much larger momentum transfer taken from the field. We illuminate the process by confronting our experimental findings of a preferred back-to-back emission of the electrons with the results of model calculations, in which the field-assisted electron recollision as well as the final-state electron-electron interaction are taken into account.

We have used the COLTRIMS technique [13] to measure in coincidence the momentum of one of the electrons and the charge state and momentum vector of the ion. The Ti:sapphire laser system produces $3 \mu \mathrm{J}$ pulses of $40 \mathrm{fs}$ at $30 \mathrm{kHz}$. The pulse is focused by a $5 \mathrm{~cm}$ focal length parabolic mirror into a precooled supersonic argon gas jet. The ions and electrons are guided by a parallel electric $(3.1 \mathrm{~V} / \mathrm{cm})$ and magnetic $(8.7 \mathrm{G})$ field onto two position sensitive channel plate detectors. The direction of polarization is chosen parallel to the gas jet. Because of the internal momentum spread of the argon atoms in the 


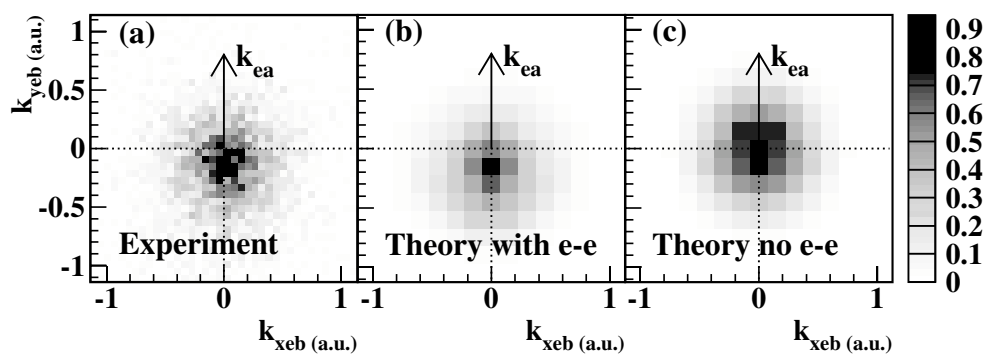

FIG. 1. Double ionization of Ar at $780 \mathrm{~nm}, 40 \mathrm{fsec}, 1.9 \times 10^{14} \mathrm{~W} / \mathrm{cm}^{2}$. Momentum distribution of electron $b$ in the plane perpendicular to the light field polarization, $\hat{\boldsymbol{\epsilon}}$. The momentum direction of electron $a$ perpendicular to $\hat{\boldsymbol{\epsilon}}$ is shown by the arrow. The data are integrated over all momentum components along $\hat{\boldsymbol{\epsilon}}$ and the magnitude of the momentum of electron $a$. (a) Experimental data (mean values $\bar{k}_{a}^{(y)}=0.37$ a.u., $\bar{k}_{b}^{(y)}=-0.18$ a.u.), (b) theoretical results, including electron-electron interaction in the final state (mean values $\bar{k}_{a}^{(y)}=0.34$ a.u., $\bar{k}_{b}^{(y)}=-0.17$ a.u.), and (c) theoretical results, neglecting electron-electron interaction. For the sake of comparison, note that the lateral momentum in single ionization of Ar is 0.256 a.u. [14].

direction of the gas jet, we could not measure the ion momentum component parallel to the polarization.

We have chosen a coordinate system $\Sigma$ where the laser polarization defines the $z$ direction, and the momentum component of electron $a$ perpendicular to the polarization defines the $y$ direction. Note that the $x$ and $y$ direction of $\Sigma$ are not fixed in the laboratory. In the laboratory frame, the electron and the ion momentum distributions are rotational symmetric around the polarization axis allowing for a precise determination of the midpoint (zero momentum). Figure 1(a) shows the $x$ and $y$ components of the momentum of electron $b\left(k_{b}^{(x)}, k_{b}^{(y)}\right)$. Note that all momenta are given Hartree atomic units [15]. By our definition of the coordinates, we have $k_{a}^{(x)}=0$, that is the perpendicular momentum of electron $a$ points upward as indicated by the arrow in Fig. 1. The data are integrated over $k_{a}^{(z)}, k_{b}^{(z)}$, and $k_{a}^{(y)}$. Clearly, the electrons $a$ and $b$ are emitted preferentially to opposite sides. We expect that this back-to-back emission indicates a strong interaction among the electrons after their double escape.

In order to elucidate the role of the final-state electronelectron interaction, we have analyzed the diagram shown in Fig. 2, in which time progresses from bottom to top. Initially, the electrons are in the ground state of the Ar atom, unperturbed of the field. At the first vertex, the laser field couples to one electron, say (1). This electron propagates in the laser field while electron (2) goes into ionic states of the $\mathrm{Ar}^{+}$ion; the intermediate state is therefore described by a product Green's function of the
Volkov states, $\{\mathbf{k}\}$, and the field-free ionic states, $\{j\}$. The two electrons then interact via the electron correlation interaction at the second vertex. This step treats electronelectron scattering in the full presence of the laser field; thus, momentum and energy can be exchanged between the electrons, and additional energy can be coupled from the field to the three-body system. Finally, the two electrons are propagated together as a pair of Coulomb interacting particles in the presence of the external field; the corresponding final-state wave function is a product of Volkov states and a Coulomb correlation function [16].

The diagram is an ad hoc extension of the leading Feynman diagram of an intense-field many-body $S$-matrix analysis of the double ionization process $[11,17]$. It includes inelastic scattering of the first electron with the second on the way out of the atom as well as on its return. At large intensities and wavelengths, the diagram may be estimated semiclassically and significant contributions can arise from the return event, which provides [5] the theoretical connection to the classical rescattering model [3]. Compared to the former $S$-matrix studies, in the model diagram the final-state wave function including the electron correlation interaction is introduced in an ad hoc way.

The diagram can be evaluated following the same steps as in earlier calculations [11,17] and the differential rate of nonsequential double ionization due to absorption of $N$ photons and emission of electrons with momenta $\mathbf{k}_{a}$ and $\mathbf{k}_{b}$ is given by (Hartree atomic units are used, $\hbar=e=m=1$ ):

$$
\frac{d W_{N}^{(N S D I)}\left(\mathbf{k}_{a}, \mathbf{k}_{b}\right)}{d \mathbf{k}_{a} d \mathbf{k}_{b}}=2 \pi \delta\left(\frac{k_{a}^{2}}{2}+\frac{k_{b}^{2}}{2}+E_{1 S}+2 U_{p}-N \omega\right)\left|T_{N}^{(N S D I)}\left(\mathbf{k}_{a}, \mathbf{k}_{b}\right)\right|^{2},
$$

with

$$
\begin{aligned}
T_{N}^{(N S D I)}\left(\mathbf{k}_{a}, \mathbf{k}_{b}\right)= & \sum_{n} \sum_{j} \int d \mathbf{k} \frac{1}{\sqrt{2}} \exp \left(-\frac{\pi \gamma_{12}}{2}\right) \Gamma\left(1+i \gamma_{12}\right) \\
& \times\left\langle\Phi^{0}\left(\mathbf{k}_{a}, \mathbf{r}_{1}\right) \Phi^{0}\left(\mathbf{k}_{b}, \mathbf{r}_{2}\right)_{1} F_{1}\left(i \gamma_{12} ; 1 ;-i\left(k_{a b} r_{12}+\mathbf{k}_{a b} \cdot \mathbf{r}_{12}\right)\right)+\mathbf{k}_{a} \leftrightarrow \mathbf{k}_{b}\left|V_{C}\right| \Phi_{j}^{+}\left(\mathbf{r}_{2}\right) \Phi^{0}\left(\mathbf{k}, \mathbf{r}_{1}\right)\right\rangle \\
& \times\left(U_{p}-n \omega\right) \frac{J_{N-n}\left[\mathbf{\alpha}_{0} \cdot\left(\mathbf{k}_{a}+\mathbf{k}_{b}-\mathbf{k}\right) ; \frac{U_{p}}{2 \omega}\right] J_{n}\left(\mathbf{\alpha}_{0} \cdot \mathbf{k} ; \frac{U_{p}}{2 \omega}\right)}{\frac{k^{2}}{2}-E_{j}+E_{0}+U_{p}-n \omega+i 0}\left\langle\Phi_{j}^{+}\left(\mathbf{r}_{2}\right) \Phi^{0}\left(\mathbf{k}, \mathbf{r}_{1}\right) \mid \Phi_{0}\left(\mathbf{r}_{1}, \mathbf{r}_{2}\right)\right\rangle .
\end{aligned}
$$




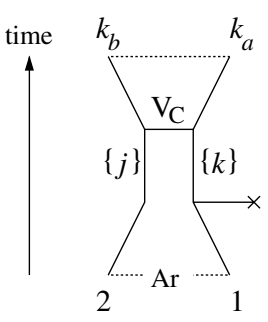

FIG. 2. Diagram of nonsequential double ionization of Ar. Note that the final-state electron-electron interaction is included, as denoted by the dotted line. For a further explanation, see the text.

$\Phi^{0}(\mathbf{k}, \mathbf{r})$ is the plane-wave state of momentum $\mathbf{k}$; analytic wave functions are used for the intermediate states $\Phi_{j}^{+}(\mathbf{r})$ of the $\mathrm{Ar}^{+}$ion and the initial ground-state wave function $\Phi_{0}\left(\mathbf{r}_{1}, \mathbf{r}_{2}\right)$ of the Ar atom [18]. $E_{0}$ and $E_{j}$ are the ground-state binding energy of Ar and the binding energy of the intermediate $\mathrm{Ar}^{+}$state, respectively. Contributions to the differential rate arising due to the $z$ components of the angular momentum in the atomic and ionic wave functions are averaged. $J_{N}(x, y)$ is the generalized Bessel function of two arguments, and $U_{p}=$ $I / 4 \omega^{2}$ and $\alpha_{0}=\sqrt{I} / \omega$ denote the quiver energy and radius of a free electron in a laser field of intensity $I$, and frequency $\omega$, respectively. $V_{C}=1 / r_{12}$ is the electron-electron correlation interaction, and $\gamma_{12}=$ $1 / 2 k_{a b}, \mathbf{k}_{a b}=\left(\mathbf{k}_{a}-\mathbf{k}_{b}\right) / 2$, and $\mathbf{r}_{12}=\mathbf{r}_{1}-\mathbf{r}_{2}$. We have restricted the radial integration in $k$ to the on-shell contributions and the sum over $j$ to the lowest ionic bound state.

Electron momentum distributions are obtained for an identical kinematical geometry and the same laser peak intensity and wavelength as in the experiment. The results for the electron momentum distribution, shown in Fig. 1(b) are found to be in very good agreement with the observations [Fig. 1(a)].

Does the back-to-back emission characteristic indicate a strong interelectron correlation among the outgoing electrons? To answer this question, in a second calculation we have deliberately neglected the final-state electron-electron interaction, which is done by setting $\gamma_{12}=0$ in Eq. (2). A comparison of the results of this calculation [Fig. 1(c)] with the results of the full calculation [Fig. 1(b)] shows that the significant back-to-back characteristics are lost, if the electron-electron repulsion in the final state is removed. This reveals that the back-toback emission does result from the final-state repulsion and not from the vertex $V_{C}$.

We have further studied the momentum distribution of electron $b$, when the perpendicular momentum of electron $a$ was fixed to a certain interval. The overall agreement between experiment [Figs. 3(a)-3(c) ] and theory [Figs. 3(d)-3(f)] is good. It is found that for small transverse momenta a symmetric distribution of the two momenta with respect to the laser polarization direction seems to dominate [Figs. 3(a) and 3(d)], while the

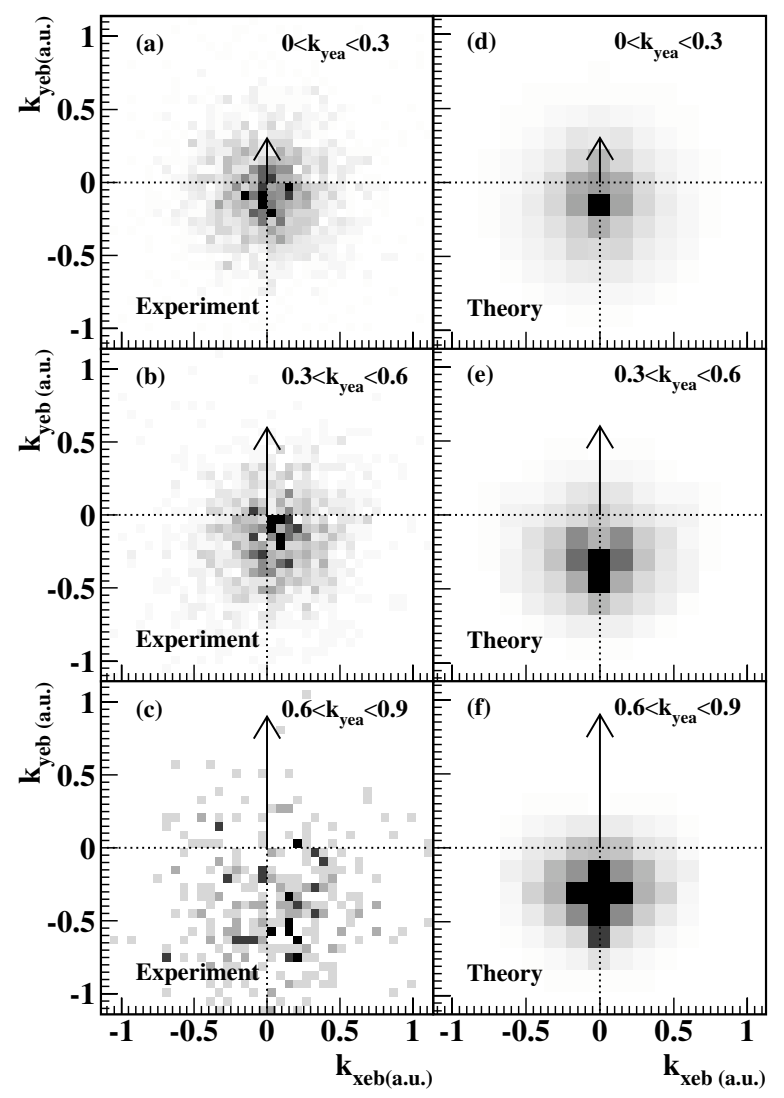

FIG. 3. Comparison between experimental data (left-hand panels) and theoretical results (right-hand panels) for the momentum distribution of electron $b$, when the transverse momentum of electron $a$ was restricted to a certain interval, (a) and (d) 0 a.u. $<k_{a}^{(x)}<0.3$ a.u., (b) and (e) 0.3 a.u. $<k_{a}^{(x)}<$ 0.6 a.u., (c) and (f) 0.6 a.u. $<k_{a}^{(x)}<0.9$ a.u. The rest is the same as in Fig. 1.

distribution gets more and more asymmetric as the transverse momentum of electron $a$ is larger.

In the present process, the trajectories of the electrons are influenced by the ion also. This is readily seen from the experimental results for the direction of the recoiling doubly charged ion, presented in Fig. 4(a). They show some back-to-back correlation to the emission of electron $a$ also. The experimental data for the ion momentum distribution can be compared with theoretical results for the distribution of the sum momentum of the two electrons, $-\left(\mathbf{k}_{a}+\mathbf{k}_{b}\right)$, since under the condition of the experiment the $\mathrm{Ar}^{2+}$ momentum, $\mathbf{k}_{\text {ion }}$, satisfies $\mathbf{k}_{\text {ion }} \approx$ $-\left(\mathbf{k}_{a}+\mathbf{k}_{b}\right)$. The back-to-back correlation is not reproduced within the present model calculations, as can be seen from the results in Fig. 4(b). The disagreement may indicate the role of further effects, which are not included in the model. In particular, the interaction between the Volkov electron and the ionic core in the intermediate state [19], and the two electrons and the ionic core in the final-state, might be essential. A scattering of the returning electron at the ionic core would lead to back-to-back momenta of electron and ion in the final state as seen in 


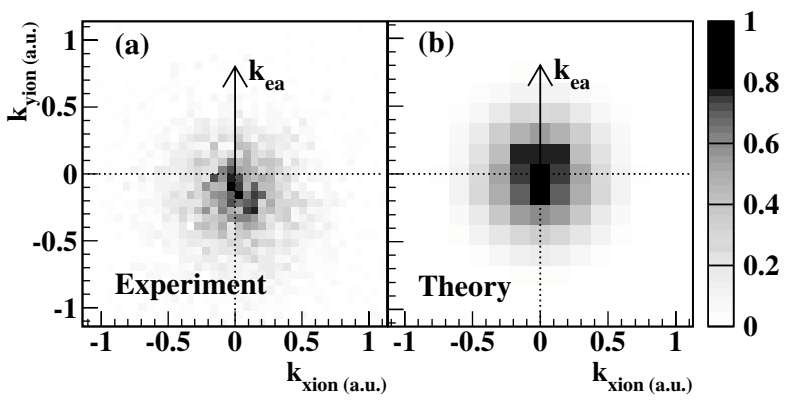

FIG. 4. Comparison between (a) experimental data and (b) theoretical results for the momentum distribution of the doubly charged ion. The rest is the same as in Fig. 1.

the data. A second effect not included in the calculation is a collisional excitation of the ion followed by field ionization [20].

In conclusion, we have presented a joint experimental and theoretical study of the transverse momentum exchange in strong field double ionization of Ar. Results of model calculations for the electron momentum distributions are found to be in very good agreement with the experimental observations. Our analysis has shown that a significant back-to-back emission of the two electrons in the plane perpendicular to the laser polarization direction is a signature of the strong electron repulsion in the final state after both electrons are in the continuum. A disagreement between experimental and theoretical results for the ion momentum might be due to electron-ion collision or collisional excitation processes, which are not included in the present theoretical model.

The Frankfurt group is indebted to Horst SchmidtBöcking for enthusiastic support of this project. A. B. acknowledges F. H. M. Faisal for indispensable support on this topic over many years. We thank A. Czasch and O. Jagutzki for their help in preparing this experiment. The experimental work is supported by DFG and BMBF.

Note added. - We note that in the meantime the role of the Coulomb correlation in the final state on the momentum components of the electrons parallel to the polarization direction of the field has been studied independently [21].
*Electronic address: abecker@mpipks-dresden.mpg.de ${ }^{\dagger}$ Electronic address: doerner@hsb.uni-frankfurt.de

[1] L. F. DiMauro and P. Agostini, Adv. At. Mol. Opt. Phys. 35, 79 (1995).

[2] R. Dörner et al., Adv. At. Mol. Opt. Phys. 48, 1 (2002).

[3] P. B. Corkum, Phys. Rev. Lett. 71, 1994 (1993).

[4] M. Yu. Kuchiev, Pis'ma Zh. Eksp. Teor. Fiz. 45, 319 (1987) [JETP Lett. 45, 404 (1987)]; J. Phys. B 28, 5093 (1995).

[5] F. H. M. Faisal and A. Becker, in Selected Topics in Electron Physics, edited by D. M. Campbell and H. Kleinpoppen (Plenum, New York, 1996), p. 397; A. Becker and F. H. M. Faisal, Opt. Ex. 8, 383 (2001).

[6] Th. Weber et al., Phys. Rev. Lett. 84, 443 (2000).

[7] R. Moshammer et al., Phys. Rev. Lett. 84, 447 (2000).

[8] Th. Weber et al., Nature (London) 405, 658 (2000).

[9] S. P. Goreslavskii, S.V. Popruzhenko, R. Kopold, and W. Becker, Phys. Rev. A 64, 053402 (2001).

[10] K. Sacha and B. Eckhardt, Phys. Rev. A 63, 043414 (2001).

[11] A. Becker and F. H. M. Faisal, Phys. Rev. Lett. 89, 193003 (2002).

[12] Li-Bin Fu, J. Liu, and S. Chen, Phys. Rev. A 65, 021406(R) (2002).

[13] R. Dörner et al., Phys. Rep. 330, 96 (2000).

[14] H. Niikura et al., Nature (London) 417, 917 (2002).

[15] 1 a.u. of momentum corresponds to $1.99288 \times$ $10^{-24} \mathrm{~kg} \mathrm{~m} \mathrm{~s}^{-1}$.

[16] F. H. M. Faisal, Phys. Lett. A 187, 180 (1994); A. Becker and F. H. M. Faisal, Phys. Rev. A 50, 3256 (1994).

[17] A. Becker and F. H. M. Faisal, J. Phys. B 29, L197 (1996); Phys. Rev. A 59, R1742 (1999); Phys. Rev. Lett. 84, 3546 (2000).

[18] E. Clementi and C. Roetti, At. Data Nucl. Data Tables 14, 177 (1974).

[19] T. Brabec, M. Yu. Ivanov, and P. B. Corkum, Phys. Rev. A 54, R2551 (1996); G. L. Yudin, and M. Yu. Ivanov, Phys. Rev. A 63, 033404 (2001); V. R. Bhardwaj et al., Phys. Rev. Lett. 86, 3522 (2001).

[20] B. Feuerstein et al., Phys. Rev. Lett. 87, 043003 (2001).

[21] C. Figueira de Morisson Faria, X. Liu, H. Schomerus, and W. Becker, arXiv.org/physics/0306143. 